\title{
GEOMETRY OF CERTAIN FIRST ORDER DIFFERENTIAL OPERATORS AND ITS APPLICATIONS TO GENERAL CONNECTIONS
}

\author{
By NaOTo ABE
}

\section{$\S 0$. Introduction.}

The covariant derivative of a connection on a vector bundle is a first order differential operator from the vector bundle to itself. In this paper, we deal with a first order differential operator from a vector bundle over a manifold to another vector bundle over the manifold whose properties are similar to those of a covariant derivative, which we will call an $O$-derivative operator. When these vector bundles are identical, the operator is a covariant derivative of a general connection in the sense of $\mathrm{T}$. Otsuki. The general connections were defined by $T$. Otsuki in [O1] as a generalized notion of usual ones. He defined the general connections on the tangent tensor bundles of a manifold and defined associating geometrical objects analogous to those of usual ones, for example, their curvature and torsion forms [O2]. In his papers [O1]-[O11], many results about general connections were obtained.

Recently the present author defined general connections on arbitrary vector bundles and studied some fundamental properties in [A]. H. Nemoto [N] applied the theory to the normal bundle of a submanifold and developed the submanifold geometry initiated by $T$. Otsuki and C.-S. Houh $[\mathrm{H}],[\mathrm{O} 10]$ and $[\mathrm{OH}]$. A. Bejancu and $\mathrm{T}$. Otsuki [BO] applied the theory to vector bundles over the tangent bundle of a manifold, especially the vertical bundle of a Finsler manifold. In these two papers, they deal with some vector bundles and linear operators from one to another. The main purpose of this paper is to generalize the theory of general connections and also prepare some formulae which can be applied to the cases treated in these papers.

In $\S 1$, we will prepare notations used in this paper and define the $O$-derivative operator. In $\S 2$, some algebraic properties of the space of $O$-derivative operators will be studied. In $\S 3$, the curvature and torsion forms of $O$-derivative operators will be defined. In $\S 4$, using given ones, we will construct $O$-derivative operators on the dual and tensor product bundles and investigate their curvature forms. In $\S 5$, we will define induced $O$-derivative operators induced by bundle maps and investigate their curvature forms. The theory will

Received October 16, 1987 
be applied to geometry of subbundles in a vector bundle with a general connection in $\S 6$ and $\S 7$.

The author would like to express his hearty thanks to Professor T. Otsuki for his helpful advice. He also would like to acknowledge the constant encouragement of Professor S. Yamaguchi.

\section{$\S 1$. Preliminaries.}

We assume that all objects are smooth and all vector bundles are real throughout this paper. Let $M$ be a manifold, $T(M)$ its tangent bundle and $C(M)$ the ring of real-valued functions on $M$. We will use letters $V, W$ and these with superscript and prime to denote vector bundles over $M$. The fibre of a vector bundle $V$ at $x \in M$ is denoted by $V_{x}$ and the dual bundle of $V$ by $V^{*}$. The space of cross-sections of $V$ is denoted by $\Gamma(V)$, which has a canonical $C(M)$-module structure. Let $\operatorname{Hom}(V, W)$ be the vector bundle of which fibre $\operatorname{Hom}(V, W)_{x}$ at $x$ is the vector space $\operatorname{Hom}\left(V_{x}, W_{x}\right)$ of linear maps from $V_{x}$ to $W_{x}$. Especially $\operatorname{Hom}(V, V)$ is denoted by $\operatorname{End}(V)$. We note that $\operatorname{Hom}(V, W)$ can be canonically identified with the tensor product $V^{*} \otimes W$. The space of vector bundle homomorphisms from $V$ to $W$ is denoted by $\operatorname{HOM}(V, W)$. We denote the zero homomorphism from $V$ to $W$ by $0_{V, W}$ or simply 0 . Especially $H O M(V, V)$, the ring of endomorphisms on $V$, is denoted by $E N D(V)$. In particular, for non-negative integer $r$, we denote the space of $W$-valued $r$-forms on $M$ by $A^{r}(M, W)$ or simply $A^{r}(W)$. We denote the identity (resp. zero) endomorphism of $V$ by $I_{V}$ (resp. $0_{V}$ or simply 0 ). We note that $H O M(V, W)$ can be canonically identified with the space $\Gamma(\operatorname{Hom}(V, W))$.

Let $H \in H O M(V, W)$ and $H_{x}=H \mid V_{x} \in H o m\left(V_{x}, W_{x}\right)$ for each $x \in M$. We define a linear map $\bar{H}: \Gamma(V) \rightarrow \Gamma(W)$ by $(\bar{H}(s))(x)=H_{x}(s(x))$ for $s \in \Gamma(V)$ and $x \in M$. Then we see that $\bar{H}$ is a $C(M)$-module homomorphism, that is, $\bar{H}\left(f_{s}\right)=$ $f \bar{H}(s)$ for $s \in \Gamma(V)$ and $f \in C(M)$. Conversely we have

LEMMA 1.1. If a linear map $B: \Gamma(V) \rightarrow \Gamma(W)$ satisfies $B(f s)=f B(s)$ for any $s \in \Gamma(V)$ and $f \in C(M)$, then there exists a unique $H \in H O M(V, W)$ such that $\bar{H}=B$.

We will use the same symbol to denote a vector bundle homomorphism $H$ and the induced linear map $\bar{H}$ on the space of cross-sections. Now we make

Definition. For $P \in H O M(V, W)$, a linear map $\nabla: \Gamma(V) \rightarrow \Gamma(T(M) * \otimes W)$ is called an $O$-derivative operator from $V$ to $W$ with the principal homomorphism $P$, if $\nabla$ satisfies

$$
\nabla f s=(d f) \otimes P s+f \nabla s \quad \text { for any } f \in C(M), s \in \Gamma(V) .
$$

Let $O(V, W ; P)$ be the set of $O$-derivative operators from $V$ to $W$ with the principal homomorphism $P$. Put $O(V, W):=\cup\{O(V, W ; P) \mid P \in H O M(V, W)\}$. Especially we denote $O(V, V ; P)$ by $O(V ; P)$ and $O(V, V)$ by $O(V)$. 
For special examples, we have the followings:

Proposition 1.2. $O\left(V, W ; 0_{V, W}\right)=H O M(V, T(M) * \otimes W)$.

This is a special case of the above lemma.

TheOREM 1.3 ([A]). $O(V ; P)$ is the set of covariant derivatives of general connections on $V$ with the principal endomorphism $P \in E N D(V)$.

COROLlaRY 1.4. $O\left(V ; I_{V}\right)$ is the set of covariant derivatives of usual connections on $V$.

Remark. In [A], using the 1 -jet bundle, it was proved that an element of $O(V ; P)$ uniquely determines a general connection defined by $\mathrm{T}$. Otsuki [O1] if $V$ is $T(M)^{*}$.

DEFinition. If $x \in M$ and $v \in T(M)_{x}$, then we define a linear map $\nabla_{v}$ : $\Gamma(V) \rightarrow W_{x}$ by $\nabla_{v} s:=i_{v}(\nabla s)$ for $s \in \Gamma(V)$, where $i_{v}$ is the inner product operator. Similarly if $X \in \Gamma(T(M))$, then we define a linear map $\nabla_{X}: \Gamma(V) \rightarrow \Gamma(W)$ by $\left(\nabla_{X} s\right)(x):=\nabla_{X(x)} s$. We call $\nabla_{X}$ the $O$-derivative operator along $X$.

Then we clearly get

Proposition 1.5. The map $T(M)_{x} \times \Gamma(V) \ni(v, s) \mapsto \nabla_{v} s \in \Gamma(W)$ is bilinear and satisfies

$$
\nabla_{v} f s=(v f)(P s)(x)+f(x) \nabla_{v} s \quad \text { for } f \in C(M) \text { and } x \in M .
$$

Moreover, we have

$$
\nabla_{X} s \in \Gamma(W) \text { and } \nabla_{f X} s=f \nabla_{X} s \text { for } s \in \Gamma(V) \text { and } X \in \Gamma(T(M)) .
$$

THEOREM 1.6. Let $U$ be an open set of $M$ and $x \in U$. For $s, s^{\prime} \in \Gamma(V)$ and $X, X^{\prime} \in \Gamma(T(M))$, if $s=s^{\prime}$ on $U$ and $X=X^{\prime}$ at $x$, then $\left(\nabla_{X} s\right)(x)=\left(\nabla_{X^{\prime}} s^{\prime}\right)(x)$.

Proof. Lemma 1.1 assures that $\left(\nabla_{X} s^{\prime}\right)(x)=\left(\nabla_{X^{\prime}} s^{\prime}\right)(x)$. It is sufficient to show that $\left(\nabla_{X} s\right)(x)=\left(\nabla_{X} s^{\prime}\right)(x)$. Take open subsets $U_{1}, U_{0}$ and $f \in C(M)$ such that $x \in U_{1}, C l\left(U_{1}\right) \subset U_{0}, C l\left(U_{0}\right) \subset U, f=1$ on $C l\left(U_{1}\right)$ and $f=0$ on $\left(U_{0}\right)^{c}$, where $C l()$ and ()$^{c}$ are the closure and the complement respectively. Then $f\left(s-s^{\prime}\right)=0$ on $M$. The above proposition implies

$$
0=\nabla_{X}\left(f\left(s-s^{\prime}\right)\right)=(X f) P\left(s-s^{\prime}\right)+f \nabla_{X}\left(s-s^{\prime}\right) .
$$

Since $(X f)(x)=0$ and $f(x)=1$, we have $\left(\nabla_{X} s-\nabla_{X} s^{\prime}\right)(x)=0$.

We note that $\nabla_{v}$ or $\nabla_{X}$ uniquely determines $\nabla$. This theorem assures that we can study $O$-derivative operators by using components with respect to local coordinates and frame fields. 
Remark. As first order differential operators, the symbols $\sigma_{1}(\nabla)$ and $\sigma_{1}\left(\nabla_{X}\right)$ for $\nabla \in O(V, W ; P)$ are

$$
\sigma_{1}(\nabla)(\xi) e=\xi \otimes P_{x} e \text { and } \sigma_{1}\left(\nabla_{X}\right)(\xi) e=\left(i_{X(x)} \xi\right) P_{x} e
$$

for $\xi \in T(M)^{*}, e \in V_{x}$ and $x \in M$.

\section{§ 2. Algebraic structures of $O(V, W)$.}

At first we consider, in $O(V, W)$, addition and multiplication by elements of $E N D(V)$ and $E N D(W)$ as follows.

Definition. If $\nabla^{i} \in O\left(V, W ; P^{i}\right)(i=1,2)$, then we define the sum $\nabla^{1}+\nabla^{2}$ by

$$
\left(\nabla^{1}+\nabla^{2}\right)_{X} s:=\nabla_{X}^{1} s+\nabla_{X}^{2} s \quad \text { for } s \in \Gamma(V) \text { and } X \in \Gamma(T(M)) .
$$

If $\nabla \in O(V, W ; P), R \in E N D(V)$ and $L \in E N D(W)$, then we define the products $L \nabla$ and $\nabla R$ by

$$
(L \nabla)_{X} s:=L\left(\nabla_{X} s\right) \text { and }(\nabla R)_{X} s:=\nabla_{X}(R s) \text {. }
$$

Proposition 2.1. $\quad \nabla^{1}+\nabla^{2} \in O\left(V, W ; P^{1}+P^{2}\right), L \nabla \in O(V, W ; L P)$ and $\nabla R \in$ $O(V, W ; P R)$. Moreover, the set $O(V, W)$ has a right $\operatorname{END}(V)$ and left $\operatorname{END}(W)$ module structure with respect to these addition and multiplication.

COROLlary 2.2 ([A]). The set $O(V)$ has a both side $E N D(V)$-module structure.

Remark. In the case of the tangent bundle, the multiplication in $O(V)$ was defined and the associative law was stated in [O7]. See also [O11] for some algebraic properties of general connections.

Furthermore, we consider multiplication by vector bundle homomorphisms.

Definition. If $L \in H O M\left(W, W^{\prime}\right), R \in H O M\left(V^{\prime}, V\right)$ and $\nabla \in O(V, W ; P)$, then we define $L \nabla$ and $\nabla R$ by

$$
\begin{aligned}
& (L \nabla)_{X} s:=L\left(\nabla_{X} s\right) \text { and }(\nabla R)_{X} t:=\nabla_{X}(R t) \\
& \quad \text { for } \quad s \in \Gamma(V), t \in \Gamma\left(V^{\prime}\right) \text { and } \quad X \in \Gamma(T(M)) .
\end{aligned}
$$

Then we have

THEOREM 2.3. $L \nabla \in O\left(V, W^{\prime} ; L P\right), \nabla R \in O\left(V^{\prime}, W ; P R\right)$ and $(L \nabla) R=L(\nabla R)$ $\in O\left(V^{\prime}, W^{\prime} ; L P R\right)$. Moreover, the maps $\operatorname{HOM}\left(W, W^{\prime}\right) \times O(V, W) \rightarrow O\left(V, W^{\prime}\right)$ and $O(V, W) \times H O M\left(V^{\prime}, V\right) \rightarrow O\left(V^{\prime}, W\right)$ are bilinear.

We will call the $O$-derivative operators $L \nabla$ and $\nabla R$ induced $O$-derivative operators induced by vector bundle homomorphisms and denote $(L \nabla) R$ by $L \nabla R$. 
When $V \cong V^{\prime}$ and $W \cong W^{\prime}$ are isomorphic vector bundles, $O(V, W)$ and $O\left(V^{\prime}, W^{\prime}\right)$ are isomorphic as modules. Then we will denote the corresponding operators by the same symbol if the isomorphisms are canonical. In the case of general connections, we get

COROLLARY 2.4 ([A]). If $\nabla \in O(V ; P), L \in H O M\left(V, V^{\prime}\right)$ and $R \in H O M\left(V^{\prime}, V\right)$, then we have $L \nabla R \in O\left(V^{\prime} ; L P R\right)$.

Remark. If $V=T(M)$ and $W$ is a subbundle of $T(M)$, this type of induced covariant derivatives of general connections was essentially defined in [O7]. In general, for $V$ and $W$, let $\bar{V}$ be their Whitney sum, $i: V \rightarrow \bar{V}$ and $i^{\prime}: W \rightarrow \bar{V}$ the inclusion homomorphisms. If $P \in H O M(V, W)$, then we can find $\bar{P} \in E N D(\bar{V})$ such that $\bar{P} i s=i^{\prime} P s$ for $s \in V$. Similarly, if $\nabla \in O(V, W)$, then we can find $\bar{\nabla} \in O(\bar{V})$ such that $\bar{\nabla} i s=i^{\prime} \nabla s$ for $s \in \Gamma(V)$, see also $\S 6$.

\section{§3. Curvature and torsion forms.}

At first we define the following form which generalizes the difference of two covariant derivatives of usual connections (see also Theorem 4.7). Let $\nabla \in$ $O(V, W ; P)$ and $\nabla^{\prime} \in O\left(V^{\prime}, V ; P^{\prime}\right)$.

Definition. If $X \in \Gamma(T(M))$, then a linear map $S\left(\nabla, \nabla^{\prime}\right)_{X}: \Gamma\left(V^{\prime}\right) \rightarrow \Gamma(W)$ is defined by

$$
S\left(\nabla, \nabla^{\prime}\right)_{X} s:=\nabla_{X}\left(P^{\prime} s\right)-P\left(\nabla_{X}^{\prime} s\right) \quad \text { for } \quad s \in \Gamma\left(V^{\prime}\right) \text {. }
$$

We call $S\left(\nabla, \nabla^{\prime}\right)$ the difference form of the pair $\left(\nabla, \nabla^{\prime}\right)$.

THEOREM 3.1. $S\left(\nabla, \nabla^{\prime}\right) \in A^{1}\left(\operatorname{Hom}\left(V^{\prime}, W\right)\right)$.

Proof. Since $\nabla P^{\prime}, P \nabla^{\prime} \in O\left(V^{\prime}, W ; P P^{\prime}\right)$, we have

$$
\nabla P^{\prime}-P \nabla^{\prime} \in O\left(V^{\prime}, W ; 0_{V^{\prime}, W}\right)=H O M\left(V^{\prime}, T(M)^{*} \otimes W\right)=A^{1}\left(H o m\left(V^{\prime}, W\right)\right) .
$$

Let ${ }^{\prime} \nabla \in O\left(W, W^{\prime} ;{ }^{\prime} P\right)$. From Theorem 2.3, it is clear that

Theorem 3.2. The map $S: O(V, W) \times O\left(V^{\prime}, V\right) \rightarrow A^{1}\left(\operatorname{Hom}\left(V^{1}, W\right)\right)$ is bilinear. Moreover, we have

and

$$
\begin{aligned}
& S\left(L \nabla, \nabla^{\prime}\right)=L S\left(\nabla, \nabla^{\prime}\right) \quad \text { for } \quad L \in H O M\left(W, W^{\prime}\right) \text {, } \\
& S\left(^{\prime} \nabla, R \nabla^{\prime}\right)=S\left({ }^{\prime} \nabla R, \nabla^{\prime}\right) \quad \text { for } \quad R \in H O M(V, W)
\end{aligned}
$$

$$
S\left({ }^{\prime} \nabla, \nabla R^{\prime}\right)=S\left({ }^{\prime} \nabla, \nabla\right) R^{\prime} \quad \text { for } \quad R^{\prime} \in H O M\left(V^{\prime}, V\right) \text {. }
$$

Corollary 3.3. $S\left({ }^{\prime} \nabla, \nabla P^{\prime}\right)-S\left({ }^{\prime} \nabla, P \nabla^{\prime}\right)+S\left({ }^{\prime} P \nabla, \nabla^{\prime}\right)=0$.

Next we define the following auxiliary operator which can be used in some 
formulae concerning curvature forms.

Definition. If $X, Y \in \Gamma(T(M))$, then a linear map $\left(\nabla \wedge \nabla^{\prime}\right)_{X, Y}: \Gamma\left(V^{\prime}\right) \rightarrow \Gamma(W)$ is defined by

$$
\left(\nabla \wedge \nabla^{\prime}\right)_{X, Y} s:=\nabla_{X}\left(\nabla_{Y}^{\prime} s\right)-\nabla_{Y}\left(\nabla_{X}^{\prime} s\right) \quad \text { for } \quad s \in \Gamma(V) .
$$

From Theorem 2.3, it is clear that

LEMMA 3.4. For $X, Y \in \Gamma(T(M)),\left(\nabla \wedge \nabla^{\prime}\right)_{X, Y}$ is bilinear on $O(V, W) \times$ $O\left(V^{\prime}, V\right)$. Moreover, we have

and

$$
\begin{array}{lll}
\left(L \nabla \wedge \nabla^{\prime}\right)_{X, Y}=L\left(\nabla \wedge \nabla^{\prime}\right)_{X, Y} & \text { for } & L \in H O M\left(W, W^{\prime}\right), \\
\left({ }^{\prime} \nabla \wedge R \nabla^{\prime}\right)_{X, Y}=\left({ }^{\prime} \nabla R \wedge \nabla^{\prime}\right)_{X, Y} & \text { for } & R \in H O M(V, W) \\
\left({ }^{\prime} \nabla \wedge \nabla R^{\prime}\right)_{X, Y}=\left({ }^{\prime} \nabla \wedge \nabla\right)_{X, Y} R^{\prime} & \text { for } & R^{\prime} \in H O M\left(V^{\prime}, V\right) .
\end{array}
$$

LEMma 3.5. For $s \in \Gamma\left(V^{\prime}\right)$ and $f \in C(M)$,

and

$$
\left(\nabla \wedge \nabla^{\prime}\right)_{f X, Y} s=f\left(\nabla \wedge \nabla^{\prime}\right)_{X, Y} s-(Y f) P \nabla_{X}^{\prime} s
$$

$$
\begin{aligned}
\left(\nabla \wedge \nabla^{\prime}\right)_{X, Y} f s= & ([X, Y] f) P P^{\prime} s-(X f) S\left(\nabla, \nabla^{\prime}\right)_{Y} s \\
& +(Y f) S\left(\nabla, \nabla^{\prime}\right)_{X} s+f\left(\nabla \wedge \nabla^{\prime}\right)_{X, Y} s .
\end{aligned}
$$

Now we define the curvature form as follows.

Definition. If $X, Y \in \Gamma(T(M))$, then a linear map $K\left({ }^{\prime} \nabla, \nabla, \nabla^{\prime}\right)_{X, Y}: \Gamma\left(V^{\prime}\right) \rightarrow$ $\Gamma\left(W^{\prime}\right)$ is defined by

$$
\begin{gathered}
K\left(\nabla^{\prime}, \nabla, \nabla^{\prime}\right)_{X, Y} s:={ }^{\prime} \nabla_{X}\left(\nabla_{Y}\left(P^{\prime} s\right)\right)-\nabla_{Y}\left(\nabla_{X}\left(P^{\prime} s\right)\right)-{ }^{\prime} P\left(\nabla_{[X, Y]}\left(P^{\prime} s\right)\right) \\
-{ }^{\prime} \nabla_{X}\left(P\left(\nabla_{Y}^{\prime} s\right)\right)+{ }^{\prime} P\left(\nabla_{X}\left(\nabla_{Y}^{\prime} s\right)\right)+{ }^{\prime} \nabla_{Y}\left(P\left(\nabla_{X}^{\prime} s\right)\right)-{ }^{\prime} P\left(\nabla_{Y}\left(\nabla_{X}^{\prime} s\right)\right)
\end{gathered}
$$

for $s \in \Gamma(V)$. We call $K\left({ }^{\prime} \nabla, \nabla, \nabla^{\prime}\right)$ the curvature form of the triple $\left({ }^{\prime} \nabla, \nabla, \nabla^{\prime}\right)$.

From the definitions, we have

LEMMA 3.6.

$$
\begin{aligned}
K\left({ }^{\prime} \nabla, \nabla, \nabla^{\prime}\right)_{X, Y} s= & \left({ }^{\prime} \nabla \wedge \nabla P^{\prime}\right)_{X, Y} s-\left({ }^{\prime} \nabla \wedge P \nabla^{\prime}\right)_{X, Y} s+\left({ }^{\prime} P \nabla \wedge \nabla^{\prime}\right)_{X, Y} s \\
& -{ }^{\prime} P \nabla_{[X, Y]} P^{\prime} s \\
= & \left({ }^{\prime} \nabla \wedge S\left(\nabla, \nabla^{\prime}\right)\right)_{X, Y} s+\left({ }^{\prime} P \nabla \wedge \nabla^{\prime}\right)_{X, Y} s-{ }^{\prime} P \nabla_{[X, Y]} P^{\prime} s \\
= & \left({ }^{\prime} \nabla \wedge \nabla P^{\prime}\right)_{X, Y} s-\left(S\left({ }^{\prime} \nabla, \nabla\right) \wedge \nabla^{\prime}\right)_{X, Y} s-{ }^{\prime} F \nabla_{[X, Y]} P^{\prime} s .
\end{aligned}
$$

From Corollary 3.3, Lemmas 1.1, 3.5 and 3.6, we get the following fundamental fact: 
THEOREM 3.7. $K\left({ }^{\prime} \nabla, \nabla, \nabla^{\prime}\right) \in A^{2}\left(\operatorname{Hom}\left(V^{\prime}, W^{\prime}\right)\right)$.

Remark. If $V=V^{\prime}=W=W^{\prime}$ and $\nabla=\nabla^{\prime}={ }^{\prime} \nabla$, then $K(\nabla, \nabla, \nabla)$ coincides with the curvature form $K(\nabla)$ of the general connection $\nabla \in O(V)$, which was defined in $[\mathrm{O} 1]$ and $[\mathrm{A}]$.

Let $\nabla^{1} \in O\left(V^{1}, W\right)$ and ${ }^{\prime} \nabla^{1} \in O\left(W^{1}, W^{\prime}\right)$. From Lemmas 3.4 and 3.6, we obtain

Theorem 3.8. The map $K: O\left(W, W^{\prime}\right) \times O(V, W) \times O\left(V^{\prime}, V\right) \rightarrow A^{2}\left(\operatorname{Hom}\left(V^{\prime}, W^{\prime}\right)\right)$ is trilinear. Moreover, we have

and

$$
\begin{array}{lll}
K\left(^{\prime} L^{\prime} \nabla, \nabla, \nabla^{\prime}\right)={ }^{\prime} L K\left({ }^{\prime} \nabla, \nabla, \nabla^{\prime}\right) & \text { for } & { }^{\prime} L \in H O M\left(W^{\prime}, W^{1}\right), \\
K\left(^{\prime} \nabla^{1}, L \nabla, \nabla^{\prime}\right)=K\left(\nabla^{1} L, \nabla, \nabla^{\prime}\right) & \text { for } & L \in H O M\left(W, W^{1}\right), \\
K\left(^{\prime} \nabla, \nabla^{1}, R \nabla^{\prime}\right)=K\left({ }^{\prime} \nabla, \nabla^{1} R, \nabla^{\prime}\right) & \text { for } & R \in H O M\left(V, V^{1}\right)
\end{array}
$$

$$
K\left({ }^{\prime} \nabla, \nabla, \nabla^{\prime} R^{\prime}\right)=K\left({ }^{\prime} \nabla, \nabla, \nabla^{\prime}\right) R^{\prime} \quad \text { for } \quad R^{\prime} \in H O M\left(V^{1}, V^{\prime}\right) .
$$

For a later section, we prepare some formulae in the following special cases:

Proposition 3.9. For $s \in \Gamma\left(V^{\prime}\right)$,

$$
\begin{aligned}
K\left({ }^{\prime} \nabla, \nabla, \nabla^{\prime}\right)_{X, Y} s & =\left({ }^{\prime} \nabla \wedge S\left(\nabla, \nabla^{\prime}\right)\right)_{X, Y} s & & (' P=0), \\
& =\left({ }^{\prime} \nabla \wedge \nabla P^{\prime}\right)_{X, Y} s+\left({ }^{\prime} P \nabla \wedge \nabla^{\prime}\right)_{X, Y} s-^{\prime} P \nabla_{[X, Y]} P^{\prime} s & & (P=0), \\
& =-\left(S\left(^{\prime} \nabla, \nabla\right) \wedge \nabla^{\prime}\right)_{X, Y} s & & \left(P^{\prime}=0\right) .
\end{aligned}
$$

COROLLARY 3.10 .

$$
\begin{aligned}
K\left({ }^{\prime} \nabla, \nabla, \nabla^{\prime}\right)_{X, Y} S & =\left({ }^{\prime} \nabla \wedge \nabla P^{\prime}\right)_{X, Y} s & & (' P=0 \text { and } P=0), \\
& =\left({ }^{\prime} P \nabla \wedge \nabla^{\prime}\right)_{X, Y} s & & \left(P=0 \text { and } P^{\prime}=0\right), \\
& =-\left({ }^{\prime} \nabla \wedge P \nabla^{\prime}\right)_{X, Y} s & & \left(' P=0 \text { and } P^{\prime}=0\right), \\
& =0 & & \left({ }^{\prime} P=0, P=0 \text { and } P^{\prime}=0\right) .
\end{aligned}
$$

We can generalize the torsion forms of usual connections on the tangent bundle to the case of $O$-derivative operators from $T(M)$ to another vector bundle over $M$ as follows. Let $\nabla \in O(T(M), W ; P)$.

Definition. For $X, Y \in \Gamma(T(M)), T(\nabla)_{X, Y} \in \Gamma(W)$ is defined by

$$
T(\nabla)_{X, Y}:=\nabla_{X} Y-\nabla_{Y} X-P([X, Y]) \text {. }
$$

We call $T(\nabla)$ the torsion form of $\nabla$. If $T(\nabla)=0$, then $\nabla$ is said to be torsionfree. 
From Lemma 1.1, we have

THEOREM 3.11. $T(\nabla) \in A^{2}(W)$.

Remark. In [O2], T. Otsuki defined the torsion form of a general connection on the tangent bundle.

THEOREM 3.12. The map $T: O(T(M), W) \rightarrow A^{2}(W)$ is linear. Moreover, for $L \in H O M\left(W, W^{\prime}\right)$, we have

$$
T(L \nabla)=L(T(\nabla))
$$

\section{$\S 4$. O-derivative operators on dual and tensor product bundles.}

If $P \in H O M(V, W)$, then $P^{*} \in H O M\left(W^{*}, V^{*}\right)$ is defined by $\left(P^{*} \eta\right)(s):=\eta(P s)$ for $\eta \in \Gamma\left(W^{*}\right)$ and $s \in \Gamma(V)$. Let $\nabla \in O(V, W ; P)$.

THEOREM 4.1. For $\eta \in \Gamma\left(W^{*}\right)$ and $X \in \Gamma(T(M))$, define a real-valued linear function $\nabla_{X}^{*} \eta$ on $\Gamma(V)$ by

$$
\left(\nabla_{X}^{*} \eta\right)(s):=X(\eta(P s))-\eta\left(\nabla_{X} s\right) \quad \text { for } \quad s \in \Gamma(V) .
$$

Then we have

(1) $\nabla_{X}^{*} \eta \in \Gamma\left(V^{*}\right)$ and $\nabla^{*} \in O\left(W^{*}, V^{*} ; P^{*}\right)$;

(2) The $\operatorname{map}^{*}: O(V, W) \rightarrow O\left(W^{*}, V^{*}\right)$ is linear;

(3) $(L \nabla)^{*}=\nabla^{*} L^{*}$ and $(\nabla R)^{*}=R^{*} \nabla^{*}$ for $L \in H O M\left(W, W^{\prime}\right), R \in H O M\left(V^{\prime}, V\right)$.

Proof. Since (2) and (3) are clear, we will prove (1). For $f \in C(M)$, we get

$$
\begin{aligned}
\left(\nabla_{X}^{*} \eta\right)(f s) & =X(f \eta(P s))-\eta\left(\nabla_{X}(f s)\right) \\
& =(X f) \eta(P s)+f X(\eta(P s))-\eta\left((X f) P s+f \nabla_{X} s\right) \\
& =f X(\eta(P s))-f \eta\left(\nabla_{X} s\right)=f\left(\nabla_{X}^{*} \eta\right)(s) .
\end{aligned}
$$

Then Lemma 1.1 implies $\nabla_{X}^{*} \eta \in \Gamma\left(V^{*}\right)$. Moreover, we get

$$
\begin{aligned}
\left(\nabla_{X} f \eta\right)(s) & =X(f \eta(P s))-f \eta\left(\nabla_{X} s\right) \\
& =(X f) \eta(P s)+f X(\eta(P s))-f \eta\left(\nabla_{X} s\right) \\
& =\left((X f) P^{*} \eta+f \nabla_{X}^{*} \eta\right)(s) .
\end{aligned}
$$

We call $\nabla^{*}$ the dual $O$-derivative operator of $\nabla$. If $P^{i} \in H O M\left(V^{i}, W^{i}\right)(i=1,2)$, then we define $P^{1} \otimes P^{2} \in H O M\left(V^{1} \otimes V^{2}, W^{1} \otimes W^{2}\right)$ by requiring $\left(P^{1} \otimes P^{2}\right)\left(s_{1} \otimes s_{2}\right):=$ $\left(P^{1} s_{1}\right) \otimes\left(P^{2} s_{2}\right)$ for $s_{i} \in \Gamma\left(V^{i}\right)$. Let $\nabla^{i} \in O\left(V^{i}, W^{i} ; P^{i}\right)(i=1,2)$.

THEOREM 4.2. We have

(1) There exists a unique $\nabla^{1} \otimes \nabla^{2} \in O\left(V^{1} \otimes V^{2}, W^{1} \otimes W^{2} ; P^{1} \otimes P^{2}\right)$ such that 


$$
\left(\nabla^{1} \otimes \nabla^{2}\right)_{X}\left(s_{1} \otimes s_{2}\right)=\left(\nabla_{X}^{1} s_{1}\right) \otimes\left(P^{2} s_{2}\right)+\left(P^{1} s_{1}\right) \otimes\left(\nabla_{X}^{2} s_{2}\right)
$$

for $s_{i} \in \Gamma\left(V^{i}\right)$ and $X \in \Gamma(T(M))$;

(2) The map $\otimes: O\left(V^{1}, W^{1}\right) \times O\left(V^{2}, W^{2}\right) \rightarrow O\left(V^{1} \otimes V^{2}, W^{1} \otimes W^{2}\right)$ is bilinear ;

(3) $\left(L^{1} \otimes L^{2}\right)\left(\nabla^{1} \otimes \nabla^{2}\right)=\left(L^{1} \nabla^{1}\right) \otimes\left(L^{2} \nabla^{2}\right)$ and $\left(\nabla^{1} \otimes \nabla^{2}\right)\left(R^{1} \otimes R^{2}\right)=\left(\nabla^{1} R^{1}\right) \otimes\left(\nabla^{2} R^{2}\right)$ for $L^{i} \in H O M\left(W^{i}, W^{i \prime}\right)$ and $R^{i} \in H O M\left(V^{i}, V^{i}\right)(i=1,2)$.

We call $\nabla^{1} \otimes \nabla^{2}$ the tensor product of $\nabla^{1}$ and $\nabla^{2}$. As in the preceeding sections, we will denote the corresponding $O$-derivative operators by the same symbol in canonical isomorphisms, for example, $V^{1} \otimes V^{2} \cong V^{2} \otimes V^{1},\left(V^{1} \otimes V^{2}\right) \otimes V^{3}$ $\cong V^{1} \otimes\left(V^{2} \otimes V^{3}\right), V^{*} \otimes W \cong \operatorname{Hom}(V, W)$ and $V^{*} \otimes V^{*} \cong(V \otimes V)^{*}$. For simplicity, we denote $\left(\otimes^{r} V\right) \otimes\left(\otimes^{s} W^{*}\right)$ by $(V, W)^{(r, s)},\left(\otimes^{r} P\right) \otimes\left(\otimes^{s} P^{*}\right) \in H O M\left((V, W)^{(r, s)}\right.$, $\left.(W, V)^{(r, s)}\right)$ by $P^{(r, s)}$ and $\left(\otimes^{r} \nabla\right) \otimes\left(\otimes^{s} \nabla^{*}\right) \in O\left((V, W)^{(r, s)},(W, V)^{(r, s)} ; P^{(r, s)}\right)$ by $\nabla^{(r, s)}$ or more simply by $\hat{\nabla}$ for $\nabla \in O(V, W)$. From the definitions, we have

Proposition 4.3. The O-derivative operator $\hat{\nabla} \in O\left((V, W)^{(r, s)},(W, V)^{(r, s)}\right.$; $\left.P^{(r, s)}\right)$ satisfies

$$
\begin{gathered}
\hat{\nabla}_{X}\left(s_{1} \otimes \cdots \otimes s_{r} \otimes \eta_{1} \otimes \cdots \otimes \eta_{s}\right)=\sum_{i=1}^{r}\left(P s_{1}\right) \otimes \cdots \otimes\left(\nabla_{X} s_{i}\right) \otimes \cdots \otimes\left(P^{*} \eta_{1}\right) \otimes \cdots \otimes\left(P^{*} \eta_{s}\right) \\
+\sum_{j=1}^{s}\left(P s_{1}\right) \otimes \cdots \otimes\left(P s_{r}\right) \otimes \cdots \otimes\left(\nabla_{X}^{*} \eta_{j}\right) \otimes \cdots \otimes\left(P^{*} \eta_{s}\right)
\end{gathered}
$$

for $s_{i} \in \Gamma(V), \eta_{j} \in \Gamma\left(W^{*}\right)$.

For a later section, we prepare

Proposition 4.4. Under the canonical isomorphisms $(V, W)^{(0,2)} \cong(W \otimes W)^{*}$ and $(W, V)^{(0,2)} \cong(V \otimes V)^{*}$, the corresponding $\hat{\nabla} \in O\left((W \otimes W)^{*},(V \otimes V)^{*}\right)$ satisfies

$$
\left(\hat{\nabla}_{X} g\right)\left(s_{1} \otimes s_{2}\right)=X\left(g\left(P s_{1} \otimes P s_{2}\right)\right)-g\left(\nabla_{X} s_{1} \otimes P s_{2}\right)-g\left(P s_{1} \otimes \nabla_{X} s_{2}\right)
$$

for $g \in \Gamma\left((W \otimes W)^{*}\right)$ and $s_{i} \in \Gamma(V)$.

Proof. Since $\eta_{1} \otimes \eta_{2} \in \Gamma\left(W^{*} \otimes W^{*}\right)$ is considered as a function on $\Gamma(W \otimes W)$ such that $\left(\eta_{1} \otimes \eta_{2}\right)\left(t_{1} \otimes t_{2}\right)=\eta_{1}\left(t_{1}\right) \eta_{2}\left(t_{2}\right)$ for $t_{1} \otimes t_{2} \in \Gamma(W \otimes W)$, we have

$$
\begin{aligned}
& \left(\hat{\nabla}_{X}\left(\eta_{1} \otimes \eta_{2}\right)\right)\left(s_{1} \otimes s_{2}\right)=\left(\left(\nabla_{X}^{*} \eta_{1}\right) \otimes\left(P^{*} \eta_{2}\right)+\left(P^{*} \eta_{1}\right) \otimes\left(\nabla_{X}^{*} \eta_{2}\right)\right)\left(s_{1} \otimes s_{2}\right) \\
& \quad=\left(\nabla_{X}^{*} \eta_{1}\right)\left(s_{1}\right)\left(P^{*} \eta_{2}\right)\left(s_{2}\right)+\left(P^{*} \eta_{1}\right)\left(s_{1}\right)\left(\nabla_{X}^{*} \eta_{2}\right)\left(s_{2}\right) \\
& \quad=\left(X\left(\eta_{1}(P s)\right)-\eta_{1}\left(\nabla_{X} s_{1}\right)\right) \eta_{2}\left(P s_{2}\right)+\eta_{1}\left(P s_{1}\right)\left(X\left(\eta_{2}\left(P s_{2}\right)\right)-\eta_{2}\left(\nabla_{X} s_{2}\right)\right. \\
& \quad=X\left(\left(\eta_{1} \otimes \eta_{2}\right)\left(P s_{1} \otimes P s_{2}\right)\right)-\left(\eta_{1} \otimes \eta_{2}\right)\left(\nabla_{X} s_{1} \otimes P s_{2}\right)-\left(\eta_{1} \otimes \eta_{2}\right)\left(P s_{1} \otimes \nabla_{X} s_{2}\right) .
\end{aligned}
$$

Proposition 4.5. Under the canonical isomorphisms $(V, W)^{(1,1)} \cong \operatorname{Hom}(W, V)$ and $(W, V)^{(1,1)} \cong \operatorname{Hom}(V, W)$, the corresponding $\hat{\nabla} \in O(\operatorname{Hom}(W, V), \operatorname{Hom}(V, W))$ satisfies 


$$
\left(\hat{\nabla}_{X} H\right) s=\nabla_{X}(H P s)-P H\left(\nabla_{X} s\right)
$$

for $H \in \Gamma(H o m(W, V))$ and $s \in \Gamma(V)$.

Proof. Since $e \otimes \eta \in \Gamma\left(V \otimes W^{*}\right)$ is considered as a function on $\Gamma(W)$ such that $(e \otimes \eta)(t)=\eta(t) e$ for $t \in \Gamma(W)$, we get

$$
\begin{aligned}
& \left(\hat{\nabla}_{X}(e \otimes \eta)\right)(s)=\left(\left(\nabla_{X} e\right) \otimes\left(P^{*} \eta\right)+(P e) \otimes\left(\nabla_{X}^{*} \eta\right)\right)(s) \\
& \quad=\left(P^{*} \eta\right)(s) \nabla_{X} e+\left(\nabla_{X}^{*} \eta\right)(s) P e=\eta(P s) \nabla_{X} e+\left(X(\eta(P s))-\eta\left(\nabla_{X} s\right)\right) P e \\
& \quad=\nabla_{X}((e \otimes \eta)(P s))-((P e) \otimes \eta)\left(\nabla_{X} s\right) .
\end{aligned}
$$

We can generalize this proposition as follows. Let $\nabla^{\prime} \in O\left(V^{\prime}, V ; P^{\prime}\right)$ and $\prime \nabla \in O\left(W, W^{\prime} ;{ }^{\prime} P\right)$. Then we have

THEOREM 4.6. Under the canonical isomorphisms $(W, V)^{(1,1)} \cong \operatorname{Hom}(V, W)$ and $\left(W^{\prime}, V^{\prime}\right)^{(1,1)} \cong \operatorname{Hom}\left(V^{\prime}, W^{\prime}\right)$, the corresponding $\quad \nabla \otimes\left(\nabla^{\prime}\right)^{*} \in O(\operatorname{Hom}(V, W)$, $\left.\operatorname{Hom}\left(V^{\prime}, W^{\prime}\right)\right)$ satisfies

$$
\left(\left(\nabla \otimes\left(\nabla^{\prime}\right)^{*}\right)_{X} H\right) s={ }^{\prime} \nabla_{X}\left(H P^{\prime} s\right)-{ }^{\prime} P H\left(\nabla_{X}^{\prime} s\right)
$$

for $H \in \Gamma(\operatorname{Hom}(V, W))$ and $s \in \Gamma\left(V^{\prime}\right)$.

The proof is similar to that of Proposition 4.5. Now we consider the difference and curvature forms $S$ and $K$. Let $\nabla^{\prime} \in O\left(V^{\prime}, V ; P^{\prime}\right)$. Theorem 4.6 implies

THEOREM 4.7. $S\left(\nabla, \nabla^{\prime}\right)=\left(\nabla \otimes\left(\nabla^{\prime}\right)^{*}\right) I_{V}$.

Remark. If $V=V^{\prime}=W$ and $\nabla=\nabla^{\prime}$, then $S(\nabla, \nabla)=\hat{\nabla} I_{V}$.

From the definitions, we have

THEOREM 4.8. For $\eta \in \Gamma\left(W^{*}\right)$ and $s \in \Gamma\left(V^{\prime}\right)$,

$$
\left(S\left(V^{\prime *}, V^{*}\right) \eta\right)(s)=\eta\left(S\left(\nabla, \nabla^{\prime}\right) s\right)=-\left(S\left(\nabla, \nabla^{\prime}\right)^{*} \eta\right)(s) .
$$

LEMMA 4.9. For $\eta \in \Gamma\left(W^{*}\right)$ and $s \in \Gamma\left(V^{\prime}\right)$,

$$
\begin{aligned}
\left(\left(\nabla^{*} \wedge \nabla^{*}\right)_{X, Y} \eta\right)(s)= & {[X, Y] \eta\left(P P^{\prime} s\right)+Y \eta\left(S\left(\nabla, \nabla^{\prime}\right)_{X} s\right) } \\
& -X \eta\left(S\left(\nabla, \nabla^{\prime}\right)_{Y} s\right)-\eta\left(\left(\nabla \wedge \nabla^{\prime}\right)_{X, Y} s\right) .
\end{aligned}
$$

Let ${ }^{\prime} \nabla \in O\left(W, W^{\prime} ;{ }^{\prime} P\right)$. From Corollary 3.3, Lemmas 3.6 and 4.9, we get

Theorem 4.10. For $\eta \in \Gamma\left(W^{\prime *}\right)$ and $s \in \Gamma\left(V^{\prime}\right)$,

$$
\left(K\left(\nabla^{*}, \nabla^{*}, \nabla^{*}\right) \eta\right)(s)=-\eta\left(K\left({ }^{\prime} \nabla, \nabla, \nabla^{\prime}\right) s\right) .
$$


Let $\nabla^{i \prime} \in O\left(\nabla^{i \prime}, \nabla^{i}\right)(i=1,2)$.

From the definitions, we have

THEOREM 4.11. For $s_{i} \in \Gamma\left(V^{i \prime}\right)(i=1,2)$,

$$
\begin{aligned}
S\left(\nabla^{1} \otimes \nabla^{2}, \nabla^{\prime \prime} \otimes \nabla^{2 \prime}\right)\left(s_{1} \otimes s_{2}\right)= & S\left(\nabla^{1}, \nabla^{1 \prime}\right) s_{1} \otimes P^{2} P^{2 \prime} s_{2} \\
& +P^{1} P^{1} s_{1} \otimes S\left(\nabla^{2}, \nabla^{2 \prime}\right) s_{2} .
\end{aligned}
$$

LEMMA 4.12. For $s_{i} \in \Gamma\left(V^{i \prime}\right)(i=1,2)$,

$$
\begin{aligned}
\left(\left(\nabla^{1} \otimes \nabla^{2}\right)\right. & \left.\wedge\left(\nabla^{\prime \prime} \otimes \nabla^{2 \prime}\right)\right)_{X, Y}\left(s_{1} \otimes s_{2}\right)=\left(\nabla^{1} \wedge \nabla^{1 \prime}\right)_{X, Y} s_{1} \otimes P^{2} P^{2 \prime} s_{2} \\
+ & S\left(\nabla^{1}, \nabla^{1 \prime}\right)_{X} s_{1} \otimes \nabla_{Y}^{2} P^{2 \prime} s_{2}-\nabla_{X}^{1} P^{1 \prime} s_{1} \otimes S\left(\nabla^{2}, \nabla^{2 \prime}\right)_{Y} s_{2} \\
& -S\left(\nabla^{1}, \nabla^{\prime \prime}\right)_{Y} s_{1} \otimes \nabla_{X}^{2} P^{2 \prime} s_{2}+\nabla_{Y}^{1} P^{1 \prime} s_{1} \otimes S\left(\nabla^{2}, \nabla^{\prime \prime}\right)_{X} s_{2} \\
& +P^{1} P^{1 \prime} s_{1} \otimes\left(\nabla^{2} \wedge \nabla^{2 \prime}\right)_{X, Y} s_{2} .
\end{aligned}
$$

Let $\nabla^{i} \in O\left(W^{i}, W^{i \prime}\right)(i=1,2)$. From Corollary 3.3, Lemmas 3.6 and 4.12 , we get

THEOREM 4.13. For $s_{i} \in \Gamma\left(V^{i \prime}\right)(i=1,2)$,

$$
\begin{aligned}
& K\left({ }^{\prime} \nabla^{1} \otimes^{\prime} \nabla^{2}, \nabla^{1} \otimes \nabla^{2}, \nabla^{\prime \prime} \otimes \nabla^{2 \prime}\right)_{X, Y}\left(s_{1} \otimes s_{2}\right)=K\left({ }^{\prime} \nabla^{1}, \nabla^{1}, \nabla^{\prime \prime}\right)_{X, Y} s_{1} \otimes{ }^{\prime} P^{2} P^{2} P^{2 \prime} s_{2} \\
& +S\left(^{\prime} \nabla^{1}, \nabla^{1} P^{1 \prime}\right)_{X} s_{1} \otimes S\left({ }^{\prime} P^{2} \nabla^{2}, \nabla^{2 \prime}\right)_{Y} s_{2}-S\left({ }^{\prime} P^{1} \nabla^{1}, \nabla^{1 \prime}\right)_{X} s_{1} \otimes S\left({ }^{\prime} \nabla^{2}, \nabla^{2} P^{2 \prime}\right)_{Y} s_{2} \\
& \left.-S\left(^{\prime} \nabla^{1}, \nabla^{1} P^{1 \prime}\right)_{Y} s_{1} \otimes S{ }^{\prime} P^{2} \nabla^{2}, \nabla^{2 \prime}\right)_{X} s_{2}+S\left({ }^{\prime} P^{1} \nabla^{1}, \nabla^{1 \prime}\right)_{Y} s_{1} \otimes S\left({ }^{\prime} \nabla^{2}, \nabla^{2} P^{2 \prime}\right)_{X} s_{2} \\
& +P^{1} P^{1} P^{1 \prime} s_{1} \otimes K\left({ }^{\prime} \nabla^{2}, \nabla^{2}, \nabla^{2 \prime}\right)_{X, Y} s_{2} .
\end{aligned}
$$

Remark. A sort of Ricci's formula for general connections on the tangent tensor bundle was obtained in [O9] and $[\mathrm{OH}]$.

\section{$\S 5$. O-derivative operators on induced bundles.}

Let $N$ be a manifold and $F: N \rightarrow M$ a map. If $V$ is a vector bundle over $M$, we denote the induced bundle over $N$ by $F^{\#} V$, the bundle map by $F: F^{\#} V$ $\rightarrow V$ and its restriction to the fibre by $F_{y}$ for $y \in N$. A linear map $F^{\#}: \Gamma(V)$ $\rightarrow \Gamma\left(F^{\#} V\right)$ is defined by $\left(F^{\#} s\right)(y):=F_{y}^{-1}(s(F(y)))$ for $s \in \Gamma(V)$ and $y \in N$. We note that there are canonical isomorphisms: $F^{\#}\left(V^{*}\right) \cong\left(F^{\#} V\right)^{*}, F^{\#}(V \otimes W) \cong\left(F^{\#} V\right) \otimes$ $\left(F^{\#} W\right)$ and $F^{\#}(\operatorname{Hom}(V, W)) \cong \operatorname{Hom}\left(F^{\#} V, F^{\#} W\right)$ for vector bundles $V$ and $W$ over $M$. For vector bundle valued forms, we make

Definition. If $H \in A^{r}(M, \operatorname{Hom}(V, W))$, we define $F^{\#} H \in A^{r}\left(N, \operatorname{Hom}\left(F^{\#} V\right.\right.$, $\left.F^{\#} W\right)$ ) by 


$$
\left(\left(F^{\#} H\right)_{y}\left(v_{1}, \cdots, v_{r}\right)\right)(t):=F_{y}^{-1}\left(\left(H_{F(y)}\left(F_{*} v_{1}, \cdots, F_{*} v_{r}\right)\right)\left(F_{y} t\right)\right)
$$

for $v_{i} \in T(N)_{y}, t \in\left(F^{\#} V\right)_{y}$ and $y \in N$, where $F_{*}$ is the differential of the map $F$ : $N \rightarrow M$.

We note that, if $P \in H O M(V, W)$, then $F^{\#} P \in H O M\left(F^{\#} V, F^{\#} W\right)$ satisfies $\left(F^{\#} P\right) F^{\#} s=F^{\#}(P s)$ for $s \in \Gamma(V)$. Let $\nabla \in O(V, W ; P)$.

THEOREM 5.1. We have

(1) There exists a unique O-derivative operator $F \# \nabla \in O\left(F^{\#} V, F \# W ; F^{\#} P\right)$ such that

$$
\left(F^{\# \nabla}\right)_{v} F^{\#} s=F_{y}^{-1}\left(\nabla_{F * v} s\right) \quad \text { for any } s \in \Gamma(V), v \in T(N)_{y}, y \in N \text {; }
$$

(2) The map $F^{\#}: O(V, W) \rightarrow O\left(F^{\#} V, F^{\#} W\right)$ is linear;

(3) $\quad F^{\#}(L \nabla)=\left(F^{\#} L\right)\left(F^{\#} \nabla\right)$ and $F^{\#}(\nabla R)=\left(F^{\#} \nabla\right)\left(F^{\#} R\right)$ for $L \in H O M\left(W, W^{\prime}\right)$ and $R \in H O M\left(V^{\prime}, V\right)$.

Proof. Since (2) and (3) are clear, we will prove (1). At first, we will define $\left(F^{\#} \nabla\right)_{v} t \in\left(F^{\#} W\right)_{y}$ for $v \in T(N)_{y}$ and $t \in \Gamma\left(F^{\#} V\right)$. Take an open set $U$ in $M$ such that $F(y) \in U$ and there exist local frame fields $e_{\alpha}(\alpha=1, \cdots, \operatorname{dim} V)$. Then $F^{\#} e_{\alpha}$ are local frame fields of $F^{\#} V$ on $F^{-1}(U)$ and $t=\sum t^{\alpha} F^{\#} e_{\alpha}$ on $F^{-1}(U)$. Now we make

$$
\left(F^{\#} \nabla\right)_{v} t:=\sum\left(v t^{\alpha}\right)\left(F^{\#}\left(P e_{\alpha}\right)\right)(y)+\sum t^{\alpha}(y) F_{y}^{-1}\left(\nabla_{F_{*} v} e_{\alpha}\right) .
$$

We can show that this definition does not depend on the choice of local frame fields on $M$. It is clear that $\left(F^{\#} \nabla\right)_{v} F^{\#} s=F_{y}^{-1}\left(\nabla_{F_{*} v} s\right)$ and satisfies the formula in Proposition 1.5. Next we will prove that $\left(F^{\#} \nabla\right)_{Y} t \in \Gamma\left(F^{\#} W\right)$ for $Y \in \Gamma(T(N))$. Take local coordinates $x^{a}(a=1, \cdots, \operatorname{dim} M)$ and $y^{i}(i=1, \cdots, \operatorname{dim} N)$ and denote $\partial_{a}=\left(\partial / \partial x^{a}\right)$ and $\partial_{i}=\left(\partial / \partial y^{i}\right)$. Since $F_{*}\left(\partial_{i}\right)_{y}=\Sigma\left(\partial_{i} x^{a}\right)(y)\left(\partial_{a}\right)_{F(y)}$, we can locally get the following formula :

$$
\left(F^{\#}\right)_{\partial_{i}} F^{\#} s=\Sigma\left(\partial_{i} x^{a}\right) F^{\#}\left(\nabla_{\partial_{a}} s\right) .
$$

Thus $\left(F^{\# \nabla}\right)_{Y} t$ is a smooth cross section of $W$. Finally Theorem 1.5 implies the statement (1).

We will call $F^{\#} \nabla$ the induced $O$-derivative operator induced by a bundle map. We note that

Corollary 5.2. On $O(V, W ; 0)$, this map $F^{\#}$ coincides with what defined for $A^{0}(M, \operatorname{Hom}(V, W))=H O M(V, W)$.

Now we consider the forms $S$ and $K$. Let $\nabla \in O\left(V^{\prime}, V\right)$. From the definitions and Theorem 5.1 and Corollary 5.2, we have

THEOREM 5.3. $S\left(F^{\# \nabla}, F^{\#} \nabla^{\prime}\right)=F^{\#}\left(S\left(\nabla, \nabla^{\prime}\right)\right)$. 
Let $' \nabla \in O\left(W, W^{\prime}\right)$. Then we have

THEOREM 5.4. $K\left(F^{\# \prime} \nabla, F^{\#} \nabla, F^{\#} \nabla^{\prime}\right)=F^{\#}\left(K\left({ }^{\prime} \nabla, \nabla, \nabla^{\prime}\right)\right)$.

Proof. Theorem 3.7 assures that it is sufficient to prove that

$$
K\left(F^{\# \prime} \nabla, F^{\#} \nabla, F^{\#} \nabla^{\prime}\right)_{\partial_{i}, \partial_{j}} F^{\#} s=F^{\#}\left(K\left({ }^{\prime} \nabla, \nabla, \nabla^{\prime}\right)_{F * \partial_{i}, F * \partial_{j}} s\right),
$$

where $\partial_{i}$ are the natural tangent frame fields as in the proof of Theorem 5.1. Using the formula in the theorem, we have

$$
\begin{aligned}
& \left(F^{\#} \nabla\right)_{\partial_{i}}\left(\left(F^{\#} \nabla\right)_{\partial_{j}} F^{\#} s\right)=\left(F^{\#} \nabla\right)_{\partial_{i}}\left(\Sigma\left(\partial_{j} x^{a}\right) F^{\#}\left(\nabla_{\partial_{a}} s\right)\right) \\
& \quad=\Sigma\left(\partial_{i}\left(\partial_{j} x^{a}\right)\right) F^{\#}\left(P \nabla_{\partial_{a}} s\right)+\Sigma\left(\partial_{i} x^{b}\right)\left(\partial_{j} x^{a}\right) F^{\#}\left(\nabla_{\partial_{b}}\left(\nabla_{\partial_{a}} s\right)\right) .
\end{aligned}
$$

Then we get

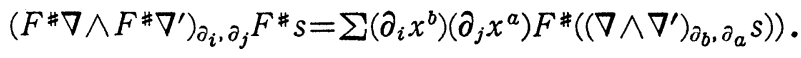

Lemma 3.6 and Theorem 5.1 imply

$$
K\left(F^{\# \prime} \nabla, F^{\# \nabla}, F^{\#} \nabla^{\prime}\right)_{\partial_{i}, \partial_{j}} F^{\#} s=\Sigma\left(\partial_{i} x^{b}\right)\left(\partial_{j} x^{a}\right) F^{\#}\left(K\left({ }^{\prime} \nabla, \nabla, \nabla^{\prime}\right)_{\partial_{b}, \partial_{a}} s\right) .
$$

Remark. Two types of induced $O$-derivative operators defined in this and the second sections can be applied to study submanifolds of spaces with a general connection, for example, see $[\mathrm{O} 10],[\mathrm{H}],[\mathrm{OH}]$ and $[\mathrm{N}]$. See also the final paragraph in the next section.

\section{$\S 6$. Induced $O$-derivative operators on subbundles.}

Let $\bar{V}$ be a vector bundle over $M, V$ a subbundle of $\bar{V}$ and $i \in H O M(V, \bar{V})$ the inclusion map. Fix a projection operator $p \in H O M(\bar{V}, V)$, that is, $p i=I_{V}$. Then we get a decomposition $\bar{V}=V \oplus V^{\prime}$ (direct sum), where $V^{\prime}$ is a subbundle of $\bar{V}$, and the inclusion $i^{\prime} \in H O M\left(V^{\prime}, \bar{V}\right)$ and the projection operator $p^{\prime} \in$ $\operatorname{HOM}\left(\bar{V}, V^{\prime}\right)$. Then we see that $p i=I_{V}, p^{\prime} i=0, p i^{\prime}=0, p^{\prime} i^{\prime}=I_{V^{\prime}}$ and $I_{\bar{V}}=i p+i^{\prime} p^{\prime}$. We note that the decomposition of $\bar{V}$ in this section may have no relations with a metric. In the next section, we will treat the decomposition induced by a metric. For $\bar{P} \in E N D(\bar{V})$, put $P:=p \bar{P} i \in E N D(V), Q:=p^{\prime} \bar{P} i \in H O M\left(V, V^{\prime}\right), Q^{\prime}:=$ $p^{\prime} \bar{P} i^{\prime} \in H O M\left(V^{\prime}, V\right)$ and $P^{\prime}:=p^{\prime} \bar{P} i^{\prime} \in E N D\left(V^{\prime}\right)$. Then we have $\bar{P} i=i P+i^{\prime} Q$ and $\bar{P} i^{\prime}=i Q^{\prime}+i^{\prime} P^{\prime}$. Let $\bar{\nabla} \in O(\bar{V} ; \bar{P})$, that is, a covariant derivative of a general connection, and put $D:=p \bar{\nabla} i, B:=p^{\prime} \bar{\nabla} i, B^{\prime}:=p \bar{\nabla} i^{\prime}$ and $D^{\prime}:=p^{\prime} \bar{\nabla} i^{\prime}$. From Proposition 2.1 and Theorem 2.3, it immediately follows that

Theorem 6.1. $D \in O(V ; P), B \in O\left(V, V^{\prime} ; Q\right), B^{\prime} \in O\left(V^{\prime}, V ; Q^{\prime}\right), D^{\prime} \in O\left(V^{\prime} ;\right.$ $\left.P^{\prime}\right)$ and

$$
\bar{\nabla} i=i D+i^{\prime} B \text { in } O(V, \bar{V}), \bar{\nabla} i^{\prime}=i B^{\prime}+i^{\prime} D^{\prime} \text { in } O\left(V^{\prime}, \bar{V}\right) \text {. }
$$

We will call $D$ (resp. $D^{\prime}$ ) the induced covariant derivative on $V$ (resp. $V^{\prime}$ ) 
and $B$ (resp. $\left.B^{\prime}\right)$ the shape operator of $V$ (resp. $V^{\prime}$ ). The first (resp. second) equality in the above theorem corresponds to Gauss' (resp. Weingarten's) formula in submanifold geometry.

Definition. If a subbundle $W$ of $\bar{V}$ satisfies $\bar{P}(W) \subset W$, then $W$ is said to be $\bar{P}$-invariant.

Since the condition that $V$ is $\bar{P}$-invariant is equivalent to $Q=0$, we get

CoRollary 6.2. If $V$ is $\bar{P}$-invariant, then $Q=0$ and $B \in O\left(V, V^{\prime} ; 0\right)=A^{1}$ $\left(\operatorname{Hom}\left(V, V^{\prime}\right)\right)$.

Theorem 6.1 shows that a general connection on $\bar{V}$ induces $O$-derivative operators on subbundles. Conversely we can obtain induced general connections on $\bar{V}$ from $O$-derivative operators as remarked in $\S 2$. For example, we see that $i O(V) p \subset O(\bar{V})$ and $i^{\prime} O\left(V, V^{\prime}\right) p \subset O(\bar{V})$ from Theorem 2.3. On the other hand, we have

Theorem 6.3. For $\bar{\nabla} \in O(\bar{V}), \bar{\nabla} \in i O(V) p$ if and only if $B=0, B^{\prime}=0$ and $D^{\prime}$ $=0$, that is,

$$
\bar{\nabla}_{X}(\Gamma(V)) \subset \Gamma(V) \text { and } \bar{\nabla}_{X}\left(\Gamma\left(V^{\prime}\right)\right)=\{0\} \quad \text { for any } X \in \Gamma(T(M)) .
$$

THEOREM 6.4. For $\bar{\nabla} \in O(\bar{V}), \bar{\nabla} \in i^{\prime} O\left(V, V^{\prime}\right) p$ if and only if $D=0, B^{\prime}=0$ and $D^{\prime}=0$, that is,

$$
\bar{\nabla}_{X}(\Gamma(V)) \subset \Gamma\left(V^{\prime}\right) \text { and } \bar{\nabla}_{X}\left(\Gamma\left(V^{\prime}\right)\right)=\{0\} \quad \text { for any } X \in \Gamma(T(M)) \text {. }
$$

Remark. It $\nabla \in O(V)$ and $\bar{\nabla}=i \nabla p \in O(\bar{V})$, then $D=p \bar{\nabla} i=\nabla$. Thus Theorems 6.1 and 6.3 generalize a theorem in $\S 3$ of [BO].

We will study the forms $S, K$ and $T$ defined in $\S 3$. Put $S(\bar{\nabla})=S(\bar{\nabla}, \bar{\nabla})$. Theorems in $\S 3,6.1$ and Corollary 6.2 imply the followings.

THEOREM 6.5 .

and

$$
\begin{aligned}
& S(D, D), S\left(B^{\prime}, B\right) \in A^{1}(\operatorname{End}(V)), S(B, D), S\left(D^{\prime}, B\right) \in A^{1}\left(\operatorname{Hom}\left(V, V^{\prime}\right)\right), \\
& S\left(D^{\prime}, D^{\prime}\right), S\left(B, B^{\prime}\right) \in A^{1}\left(\operatorname{End}\left(V^{\prime}\right)\right), S\left(B^{\prime}, D^{\prime}\right), S\left(D, B^{\prime}\right) \in A^{1}\left(\operatorname{Hom}\left(V^{\prime}, V\right)\right)
\end{aligned}
$$

$$
\begin{aligned}
& p S(\bar{\nabla}) i=S(D, D)+S\left(B^{\prime}, B\right), p^{\prime} S(\bar{\nabla}) i=S(B, D)+S\left(D^{\prime}, B\right), \\
& p^{\prime} S(\bar{\nabla}) i^{\prime}=S\left(D^{\prime}, D^{\prime}\right)+S\left(B, B^{\prime}\right), p S(\bar{\nabla}) i^{\prime}=S\left(B^{\prime}, D^{\prime}\right)+S\left(D, B^{\prime}\right) .
\end{aligned}
$$

Proposition 6.6. If $V$ is $\bar{P}$-invariant, then $S(B, D)=B P, S\left(D^{\prime}, B\right)=-P^{\prime} B$, $S\left(B^{\prime}, B\right)=-Q^{\prime} B$ and $S\left(B, B^{\prime}\right)=B Q^{\prime}$.

COROLlary 6.7. If $V$ and $V^{\prime}$ are $\bar{P}$-invariant, then 
and

$$
S(D, D)=S(D), S\left(B^{\prime}, B\right)=0, S(B, D)=B P, S\left(D^{\prime}, B\right)=-P^{\prime} B
$$

$$
p S(\bar{\nabla}) i=S(D), p^{\prime} S(\bar{\nabla}) i=B P-P^{\prime} B .
$$

We have also equations for $V^{\prime}, V$ instead of $V, V^{\prime}$.

Put $K(\bar{\nabla})=K(\bar{\nabla}, \bar{\nabla}, \bar{\nabla})$. The theorems in $\S 3$ imply

\section{THEOREM 6.8 .}

$K(D, D, D), K\left(B^{\prime}, B, D\right), K\left(B^{\prime}, D^{\prime}, B\right), K\left(D, B^{\prime}, B\right) \in A^{2}(\operatorname{End}(V))$,

$K(B, D, D), K\left(D^{\prime}, B, D\right), K\left(D^{\prime}, D^{\prime}, B\right), K\left(B, B^{\prime}, B\right) \in A^{2}\left(H o m\left(V, V^{\prime}\right)\right)$,

$K\left(D^{\prime}, D^{\prime}, D^{\prime}\right), K\left(B, B^{\prime}, D^{\prime}\right), K\left(B, D, B^{\prime}\right), K\left(D^{\prime}, B, B^{\prime}\right) \in A^{2}\left(\operatorname{End}\left(V^{\prime}\right)\right)$,

$K\left(B^{\prime}, D^{\prime}, D^{\prime}\right), K\left(D, B^{\prime}, D^{\prime}\right), K\left(D, D, B^{\prime}\right), K\left(B^{\prime}, B, B^{\prime}\right) \in A^{2}\left(H o m\left(V^{\prime}, V\right)\right)$

and

$$
\begin{aligned}
& p K(\bar{\nabla}) i=K(D, D, D)+K\left(B^{\prime}, B, D\right)+K\left(B^{\prime}, D^{\prime}, B\right)+K\left(D, B^{\prime}, B\right), \\
& p^{\prime} K(\bar{\nabla}) i=K(B, D, D)+K\left(D^{\prime}, B, D\right)+K\left(D^{\prime}, D^{\prime}, B\right)+K\left(B, B^{\prime}, B\right), \\
& p^{\prime} K(\bar{\nabla}) i^{\prime}=K\left(D^{\prime}, D^{\prime}, D^{\prime}\right)+K\left(B, B^{\prime}, D^{\prime}\right)+K\left(B, D, B^{\prime}\right)+K\left(D^{\prime}, B, B^{\prime}\right), \\
& p K(\bar{\nabla}) i^{\prime}=K\left(B^{\prime}, D^{\prime}, D^{\prime}\right)+K\left(D, B^{\prime}, D^{\prime}\right)+K\left(D, D, B^{\prime}\right)+K\left(B^{\prime}, B, B^{\prime}\right) .
\end{aligned}
$$

Proof. From Theorems 3.8 and 6.1, we have

$$
\begin{aligned}
K(\bar{\nabla}, \bar{\nabla}, \bar{\nabla}) i & =K(\bar{\nabla}, \bar{\nabla}, \bar{\nabla} i)=K\left(\bar{\nabla}, \bar{\nabla}, i D+i^{\prime} B\right)=K(\bar{\nabla}, \bar{\nabla}, i D)+K\left(\bar{\nabla}, \bar{\nabla}, i^{\prime} B\right) \\
& =K(\bar{\nabla}, \bar{\nabla} i, D)+K\left(\bar{\nabla}, \bar{\nabla} i^{\prime}, B\right) .
\end{aligned}
$$

After some steps, we have

$$
\begin{aligned}
K(\bar{\nabla}) i= & i\left(K(D, D, D)+K\left(B^{\prime}, B, D\right)+K\left(B^{\prime}, D^{\prime}, B\right)+K\left(D, B^{\prime}, D\right)\right) \\
& +i^{\prime}\left(K(B, D, D)+K\left(D^{\prime}, B, D\right)+K\left(D^{\prime}, D^{\prime}, B\right)+K\left(B, B^{\prime}, B\right)\right) .
\end{aligned}
$$

Thus the first two formulae hold. The rest can be proved by the same method.

The first three equations in this theorem correspond to the equations of Gauss, Codazzi and Ricci respectively in submanifold geometry.

Remark. If $\nabla \in O(V)$ and $\bar{\nabla}=i \nabla p \in O(\bar{V})$, then $K(\bar{\nabla}) i=i K(\nabla)$. Thus Theorem 6.8 generalizes a theorem in $\S 3$ of $[\mathrm{BO}]$.

From Proposition 3.9 and Corollary 3.10, we get

Theorem 6.9. If $V$ is $\bar{P}$-invariant, then

$$
K\left(B^{\prime}, B, D\right)_{X, Y}=\left(B^{\prime} \wedge B P\right)_{X, Y}+\left(Q^{\prime} B \wedge D\right)_{X, Y}-Q^{\prime} B_{[X, Y]} P,
$$


and

$$
\begin{aligned}
& K\left(B^{\prime}, D^{\prime}, B\right)=-\left(S\left(B^{\prime}, D^{\prime}\right) \wedge B\right), \\
& K\left(D, B^{\prime} B\right)=-\left(S\left(D, B^{\prime}\right) \wedge B\right), \\
& K(B, D, D)=(B \wedge S(D, D)), \\
& K\left(D^{\prime}, B, D\right)_{X, Y}=\left(D^{\prime} \wedge B P\right)_{X, Y}+\left(P^{\prime} B \wedge D\right)_{X, Y}-P^{\prime} B_{[X, Y]} P, \\
& K\left(D^{\prime}, D^{\prime}, B\right)=-\left(S\left(D^{\prime}, D^{\prime}\right) \wedge B\right), \\
& K\left(B, B^{\prime}, B\right)=-\left(B \wedge Q^{\prime} B\right), \\
& K\left(B, B^{\prime}, D^{\prime}\right)=\left(B \wedge S\left(B^{\prime}, D^{\prime}\right)\right), \\
& K\left(B, D, B^{\prime}\right)=\left(B \wedge S\left(D, B^{\prime}\right)\right), \\
& K\left(D^{\prime}, B, B^{\prime}\right)_{X, Y}=\left(D^{\prime} \wedge B Q^{\prime}\right)_{X, Y}+\left(P^{\prime} B \wedge B^{\prime}\right)_{X, Y}-P^{\prime} B_{[X, Y]} Q^{\prime} \\
& K\left(B^{\prime}, B, B^{\prime}\right)_{X, Y}=\left(B^{\prime} \wedge B Q^{\prime}\right)_{X, Y}+\left(Q^{\prime} B \wedge B^{\prime}\right)_{X, Y}-Q^{\prime} B_{[X, Y]} Q^{\prime},
\end{aligned}
$$

where $X, Y \in \Gamma(T(M))$.

COROllary 6.10. If $V$ and $V^{\prime}$ are $\bar{P}$-invariant, then

$$
\begin{aligned}
& K(D, D, D)=K(D), K\left(B^{\prime}, B, D\right)=\left(B^{\prime} \wedge B P\right), \\
& K\left(B^{\prime}, D^{\prime}, B\right)=-\left(B^{\prime} \wedge P^{\prime} B\right), K\left(D, B^{\prime}, B\right)=\left(P B^{\prime} \wedge B\right), \\
& K(B, D, D)=(B \wedge S(D, D)), \\
& K\left(D^{\prime}, B, D\right)_{X, Y}=\left(D^{\prime} \wedge B P\right)_{X, Y}+\left(P^{\prime} B \wedge D\right)_{X, Y}-P^{\prime} B_{[X, Y]} P, \\
& K\left(D^{\prime}, D^{\prime}, B\right)=-\left(S\left(D^{\prime}, D^{\prime}\right) \wedge B\right), K\left(B, B^{\prime}, B\right)=0,
\end{aligned}
$$

and

$$
\begin{aligned}
& p K(\bar{\nabla}) i=K(D)+\left(B^{\prime} \wedge B P\right)-\left(B^{\prime} \wedge P^{\prime} B\right)+\left(P B^{\prime} \wedge B\right) \\
& p^{\prime} K(\bar{\nabla})_{X, Y} i=(B \wedge S(D, D))_{X, Y}+\left(D^{\prime} \wedge B P\right)_{X, Y}+\left(P^{\prime} B \wedge D\right)_{X, Y} \\
& \quad-P^{\prime} B_{[X, Y]} P-\left(S\left(D^{\prime}, D^{\prime}\right) \wedge B\right)_{X, Y} .
\end{aligned}
$$

We have also equations for $V, V^{\prime}$ instead of $V, V^{\prime}$.

Let $M$ be a submanifold of a manifold $\bar{M}$ and $j: M \rightarrow \bar{M}$ the inclusion. Put $\bar{V}=j^{\#} T(\bar{M})=T(\bar{M}) \mid M, V=T(M)$ and let $V^{\prime}$ be a complementary subbundle of $V$ in $\bar{V}$. For $\nabla \in O(T(\bar{M}))$, put $\bar{\nabla}=j^{\#} \nabla \in O(\bar{V})$. Then the above facts can be applied to this case. Furthermore we have the following results on torsion forms.

THEOREM 6.11.

and

$$
T(D) \in A^{2}(T(M)), T(B) \in A^{2}\left(V^{\prime}\right)
$$




$$
j^{\#}(T(\nabla))=i T(D)+i^{\prime} T(B) .
$$

COROLlaRY 6.12. If $\nabla$ is torsion-free, then $D$ and $B$ are torsion-free.

Proposition 6.13. If $T(M)$ is $\bar{P}$-invariant, then

$$
T(B)_{X, Y}=B_{X} Y-B_{Y} X \quad \text { for } \quad X, Y \in \Gamma(T(M)) \text {. }
$$

COROLlaRY 6.14. If $T(M)$ is $\bar{P}$-invariant, then $j \#(\nabla)=i T(D)$ if and only if $B$ is symmetric, that is,

$$
B_{X} Y=B_{Y} X \quad \text { for any } X, Y \in \Gamma(T(M)) .
$$

Remark. In the case of an adapted submanifold, that is, $V$ and $V^{\prime}$ are $\bar{P}$ invariant, Corollaries $6.2,6.10,6.12$ and 6.14 were partially obtained in [H], $[\mathrm{O} 10]$ and $[\mathrm{N}]$.

\section{$\S 7$. Metric general connections and subbundles.}

At first we make

Definition. If $g \in \Gamma\left((W \otimes W)^{*}\right)$ is symmetric and non-degenerate on each fiber of $W$, then $g$ is called a metric on $W$. If $\nabla \in O(V, W)$ satisfies $\hat{\nabla} g=0$ (see Proposition 4.4), then $\nabla$ is said to be metrical with respect to $g$.

From now on, we use the same vector bundles, homomorphisms and $O$ derivative operators as those in the first paragraph in $\S 6$. Let $\bar{g}$ be a metric on $\bar{V}$ and assume that $g:=\bar{g}(i \otimes i) \in \Gamma\left((V \otimes V)^{*}\right)$ and $g^{\prime}:=\bar{g}\left(i^{\prime} \otimes i^{\prime}\right) \in \Gamma\left(\left(V^{\prime} \otimes V^{\prime}\right)^{*}\right)$ are also metrics on $V$ and $V^{\prime}$ respectively.

Definition. If $\bar{g}\left(i \otimes i^{\prime}\right)=0$, that is, $\bar{g}=g(p \otimes p)+g^{\prime}\left(p^{\prime} \otimes p^{\prime}\right)$, then $\bar{g}$ is said to be compatible with the decomposition of $\bar{V}$ by $V$ and $V^{\prime}$.

Recall that we use the following notations:

$$
\hat{\bar{\nabla}} \bar{g}=(\bar{\nabla} \otimes \bar{\nabla})^{*} \bar{g}, \hat{D} g=(D \otimes D)^{*} g \text { and } \hat{B} g^{\prime}=(B \otimes B)^{*} g^{\prime} \text {. }
$$

THEOREM 7.1. If $g$ is compatible with the decomposition, then we have

$$
\begin{aligned}
& (\hat{\bar{\nabla}} \bar{g})(i \otimes i)=\hat{D} g+\hat{B} g^{\prime} \quad \text { in } \quad \Gamma\left(T(M)^{*} \otimes(V \otimes V)^{*}\right), \\
& (\hat{\bar{\nabla}} \bar{g})\left(i \otimes i^{\prime}\right)=\left(D \otimes B^{\prime}\right)^{*} g+\left(B \otimes D^{\prime}\right)^{*} g^{\prime} \text { in } \Gamma\left(T(M)^{*} \otimes\left(V \otimes V^{\prime}\right)^{*}\right)
\end{aligned}
$$

and also equations for $V^{\prime}, V$ instead of $V, V^{\prime}$.

Proof. From Theorems 4.1, 4.2 and 6.1, we have 


$$
\begin{aligned}
& \left((\bar{\nabla} \otimes \bar{\nabla})^{*} \bar{g}\right)(i \otimes i)=(i \otimes i) *((\bar{\nabla} \otimes \bar{\nabla}) * \bar{g})=((\bar{\nabla} \otimes \bar{\nabla})(i \otimes i))^{*} \bar{g} \\
= & (\bar{\nabla} i \otimes \bar{\nabla} i)^{*} \bar{g}=\left(\left(i D+i^{\prime} B\right) \otimes\left(i D+i^{\prime} B\right)\right)^{*} \bar{g} \\
= & ((i \otimes i)(D \otimes D))^{*} \bar{g}+\left(\left(i \otimes i^{\prime}\right)(D \otimes B)\right)^{*} \bar{g}+\left(\left(i^{\prime} \otimes i\right)(B \otimes D)\right)^{*} \bar{g}+\left(\left(i^{\prime} \otimes i^{\prime}\right)(B \otimes B)\right)^{*} \bar{g} \\
= & (D \otimes D)^{*} g+(D \otimes B)^{*}\left(\left(i \otimes i^{\prime}\right)^{*} \bar{g}\right)+(B \otimes D)^{*}\left(\left(i^{\prime} \otimes i\right)^{*} \bar{g}\right)+(B \otimes B)^{*} g^{\prime} .
\end{aligned}
$$

The second formula follows similarly.

From Proposition 4.4 and Corollary 6.2, we get

Theorem 7.2. If $V$ is $\bar{P}$-invariant, then

and

$$
\hat{B}_{X} g^{\prime}=0,\left(B \otimes D^{\prime}\right)_{X}^{*} g^{\prime}=-g^{\prime}\left(B_{X} \otimes P^{\prime}\right)
$$

$$
\left(D^{\prime} \otimes B\right)_{X}^{*} g^{\prime}=-g^{\prime}\left(P^{\prime} \otimes B_{X}\right) \quad \text { for } \quad X \in \Gamma(T(M)) \text {. }
$$

COROLlary 7.3. If $g$ is compatible with the decomposition and $V$ and $V^{\prime}$ are $\bar{P}$-invariant, then we have

$$
\left(\hat{\bar{\nabla}}_{X} \bar{g}\right)(i \otimes i)=\hat{D}_{X} g,\left(\hat{\nabla}_{X} \bar{g}\right)\left(i \otimes i^{\prime}\right)=-g\left(P \otimes B_{X}^{\prime}\right)-g^{\prime}\left(B_{X} \otimes P^{\prime}\right)
$$

and also equations for $V^{\prime}, V$ instead of $V, V^{\prime}$.

COROLLARY 7.4. If $\bar{g}$ is compatible wite the decomposition and $V$ and $V^{\prime}$ are $\bar{P}$-invariant, then $\bar{\nabla}$ is metrical if and only if $D$ and $D^{\prime}$ are metrical and $g(P \otimes$ $\left.B_{X}^{\prime}\right)=-g^{\prime}\left(B_{X} \otimes P^{\prime}\right)$ for any $X \in \Gamma(T(M))$.

Remark. In the case of an adapted submanifold, Corollary 7.4 was partially obtained in $[\mathrm{H}]$ and $[\mathrm{N}]$.

\section{REFERENCES}

[A] Abe, N., General connections on vector bundles, Kodai Math. J. 8 (1985), 322-329.

[B O] Bejancu, A. and Otsuki, T., General Finsler connections on a Finsler vector bundle, Kodai Math. J. 10 (1987), 143-452.

[H] HouH, C.S., Submanifolds in a Riemannian manifold with general connections, Math. J. Okayama Univ. 12 (1963), 1-37.

[N] Nemoto, H., On differential geometry of general connections, TRU Math. 21 (1985), 67-94.

[O1] Otsuki, T., Tangent bundles of order 2 and general connections, Math. J. Okayama Univ. 8 (1958), 143-180.

[O2] OTsUKI, T., On general connections I, Math. J. Okayama Univ. 9 (1960), 99-164.

[O3] OтsuKI, T., On general connections II, Math. J. Okayama Univ. 10 (1961), 113124.

[04] Otsuki, T., On normal general connections, Kodai Math. Sem. Rep. 13 (1961), $152-166$. 
[05] Otsuki, T., On metric general connections, Proc. Japan Acad. 37 (1962), 183-188.

[06] Otsuki, T., A note on metric general connections, Proc. Japan Acad. 38 (1962), 409-413.

[O7] Otsuki, T., General connections $A \Gamma A$ and the parallelism of Levi-Civita, Kodai Math. Sem. Rep. 14 (1962), 40-52.

[08] OTsuki, T., On basic curves in spaces with normal general connections, Kodai Math. Sem. Rep. 14 (1962), 110-118.

[09] OTsuki, T., On curvatures of spaces with normal general connections I, Kodai Math. Sem. Rep. 15 (1963), 52-61.

[O10] OтsuKI, T., On curvatures of spaces with normal general connections II, Kodai Math. Sem. Rep. 15 (1963), 184-194.

[011] Otsuki, T., A note on general connections, Proc. Japan Acad. 41 (1965), 6-10.

[OH] OTsuki, T. AND Houn, C.S., Ricci's formula for normal general connections and its applications, Kodai Math. Sem. Rep. 17 (1965), 74-84.

Department of Mathematics

FACUlTY OF SCIENCE

SCIENCE UNIVERSITY OF TOKYO

TOKYO, JAPAN 162 